\title{
A novel process to create drugs for cancer prevention and treatment by modern molecular technology
}

\begin{abstract}
This article reported, in order to prevent and treat cancer well, a breakthrough way of creating new drugs has been found. With new modern molecular research technology, the genome-wide association study and the carcinogenesis theory of gene multiple hits, the breakthrough way of creating new drugs has been built by imperatively motive to control the cancer. The genome-wide association study has emerged as a powerful and successful tool to identify common disease alleles, including esophageal squamous cell carcinoma and other cancers. These research projects and results by genome-wide association study have further proved that genome-wide association and genome-wide gene-environment interaction of cancer has more than two susceptibility loci of genome association or interaction of cancer. The research project and its results have further supported the new theory of carcinogenesis, the carcinogenesis theory of gene multiple hits.

The gene susceptibility loci of cancer may be caused by environmental agents, chronic infection, et al., including alcohol drinking. The risk factors attributable fraction of susceptibility loci could be calculated. The risk factors attributable fraction of susceptibility loci means how much every risk factor of cancer contributing on the cause of susceptibility loci. So we should create the new way to prevent or treat the cancer from the view points of the genetic etiology of multiple hits and susceptibility loci by creating new drugs of the isomeride or changed structure of cancer risk factors.

Preventing cancer caused by occupational agents and environmental agents could result in possible creation of drugs of the isomeride or changed structure of cancer risk factors. Preventing cancer caused by the tobacco smoking, alcohol drinking, infectious agents and other carcinogens including physical factors could also result in possible creation of drugs after genome-wide association analyses of the cancer to identify susceptibility loci and the gene-risk factors interactions have been done and have found their interaction pathways when they are the highest risk factors of cancer attributable fraction of susceptibility loci. Then when the structures of the risk factors of cancer, their interaction pathways with genes, gene susceptibility loci of cancer were clearly proved. The isomeride or changed structure of cancer risk factors, this is the blocker, inhibitors or the blocking agent of the cancer risk factors to prevent the carcinogenesis of cancer risk factors, could be created as new drugs. The strategy and the new concept to create new drugs as the blocker, inhibitors or the blocking agent of the cancer risk factors for preventing and treating cancer by modern cancer molecular research technology, genome-wide association study and the carcinogenesis theory of gene multiple hits, could have great value to pursue and research further.
\end{abstract}

Keywords: genome-wide association study, carcinogenesis theory, environmental agents, cancer susceptibility loci, drug creation, cancer prevention

\section{Introduction}

Up to now, cancer control worldwide has not reached significant result. Now, cancers have been changing into the common diseases, from rare diseases. Cancer prevention and treatment have been still being the striving traditional methods to control the cancer devasting diseases which have not had the satisfied treatment results in most kinds of cancers. So the cancer prevention has been being paid attention heavily by international cancer societies. Among the methods of cancer prevention, the chemical intervention has been used by some research groups. But no significant results have been found. To say nothing the chemical intervention was accepted by international societies. The main reason of failing cancer chemical intervention is that the chemical subjects have not selected rightly
Volume 7 Issue I - 2019

\author{
Xu Han-You \\ Department of High Dependency Unit, Tongxiong Oriental \\ Hospital, China
}

\author{
Correspondence: Xu Han-You, Department of High \\ Dependency Unit, Tongxiong Oriental Hospital, Zhejiang \\ Province, China, Tel 0086-I55I896253I, \\ Emailabc|3579-you@I26.com
}

Received: January 23, 2019 | Published: February 22, 2019 and the cancer etiology and the carcinogenesis have not been rightly uncovered.

Up to now, a new molecular research technology, the genome-wide association study (GWAS) has emerged as a powerful and successful tool to identify common disease alleles, including esophageal squamous cell carcinoma and other cancers. The carcinogenesis theory of gene multiple hits has been accepted by international cancer societies. And lots of risk factors of cancer as human carcinogens have been proved and genome-wide gene-environment interaction analysis of cancer have been emerging. All which have paved the way to create the effective drugs to intervention prevent and treat or cure the cancer. So this article is to report a novel process to create drugs for cancer prevention and treatment by modern molecular technology. 
The modern cancer molecular technologies and their usefulness to create drugs for cancer intervention, prevention and treatment or cure

The technology of genome-wide association study and the genome-wide gene-environment interaction analysis

As we have known. GWAS has emerged as a powerful and successful tool to identify common disease alleles, including esophageal squamous cell carcinoma. So far, GWAS of cancers of the upper aerodigestive tract, including ESCC in individuals of European $^{1,2}$ and Japanese ancestry, ${ }^{3}$ have shown that variants in $\mathrm{ADH}$ genes and/or ALDH2 are associated with risk of ESCC; in addition, these studies have shown an interaction for these loci with alcohol. Two GWAS showed that variants in PLCE1 and, perhaps, C20orf54 are associated with risk of ESCC in Chinese populations. ${ }^{4,5}$ Interestingly and hopefully, more and more genome-wide association study and a genome-wide gene-environment interaction analysis of cancer have been emerging. The $\mathrm{Chen} \mathrm{Wu}$, et al.[6] have found that genome-wide association analyses of esophageal squamous cell carcinoma in Chinese identify multiple susceptibility loci and geneenvironment interactions. These research projects and results have further proved that genome-wide association and genome-wide geneenvironment interaction of cancer has more than two susceptibility loci of genome association or interaction of cancer.

\section{The carcinogenesis theory of gene multiple hits}

Lots of molecular researches have implicated a set of genetic variations that confer susceptibility to esophageal squamous cell carcinoma (ESCC), primarily variations that are related to alcohol metabolism..$^{7-12}$ The results underscore the direct genetic contribution to ESCC risk, and the genetic contribution to ESCC through interaction with alcohol consumption. The GWAS research and its results have further supported the theory of carcinogenesis that X. Han-You has found, ${ }^{13}$ the carcinogenesis theory of gene multiple hits. The summery of carcinogenesis theory of gene multiple hits as follow: We have known that cancer development is caused by long time effecting of carcinogens. The carcinogens include environmental or chemical factors; biological factors; physical factor factors of psychological, social, behavioral, emotional and spiritual issues and hereditary factors, et al. All these kinds of carcinogens display complicated effecting on human body in long time. At the final stage, the cancer is developed. All these complicated, lots kinds of carcinogens, through many different ways, finally damage many different genes on the chromosomes which lead to develop cancer.

The present molecular biological researches indicate that the cancer development involves two groups' genes which contain two different ways of patho-physiology. One way is that many different carcinogens, through different ways, damages and effects the same or (and) different proto-oncogenes. The proto-oncogenes are hit by many different carcinogens. Then become oncogenes by molecular biological changes. The oncogenes, by different ways, promote or enhance the wrong cell proliferation and finally cause carcinogenesis. At the same, the other way of patho-physiology caused by many different carcinogens is that many different carcinogens, by different ways, damage and effect the same or (and) different tumor suppressive genes or anti-oncogenes. This makes the tumor suppressive genes dysfunction or losing their functions. The dysfunctioned tumor suppressive genes, by different ways, promote or enhance the wrong cell proliferation and finally cause carcinogenesis. The oncogenes and tumor suppressive genes or anti-oncogenes work wrongly and promote or enhance the wrong cell proliferation constantly. After all, we may say in this way that before the cancer development, there are many different proto-oncogenes and tumor suppressive genes suffering from many different hits and damages by many carcinogens. That is gene multiple hits. And finally, after the genes are hit many times by the carcinogens of environmental or chemical factors; biological factors; physical factors and hereditary factors, et al. The cancer is developed.

Apart from the lots of present molecular research findings, there are lots of other research findings which had published before the GWAS technology emerged also support the gene multiple hits theory of carcinogenesis.

The major previous theory for mechanism of cancer development is the theory of two hits of gene. ${ }^{14}$ It is clear that this old theory could not fully explain all the findings for mechanism of cancer development. Only the theory-of-gene-multiple-hits can explain fully all the scientific findings for the mechanism of cancer development, the carcinogenesis.

\section{Definition of exposure of the risk factors of cancer}

Up to now, usually the definition of exposure of the risk factors of cancer has been based on the including criteria:

a. Agents that have sufficient evidence as human carcinogens according to International Agency for Research on Cancer (IARC) Monographs. ${ }^{15}$

b. Agents are causally associated with a reduced risk of cancer, according to IARC Cancer Prevention Handbook. ${ }^{16}$

c. Agents that have never been evaluated by IARC, but with strong evidence for a causal association with cancer risk (e.g. reproductive factors and breast cancer risk). ${ }^{17}$

But in the nature, there are agents or chemicals have been producing newly which may have cancer risk. And also, there are others or unknown factors which may have cancer risk. Nevertheless, the agents or factors defined by IARC as exposure of the risk factors of cancer are main risk factors of cancer at present. Furthermore, definition of exposure of the risk factors of cancer by IARC has been being continued. Therefore, all the structures and the mechanism of the risk factors of cancer could be defined.

\section{The mechanism of the novel process to create drugs for cancer prevention and treatment by modern molecular technology}

The GWAS researches of cancer have conducted genome-wide association study and genome-wide gene-environment interaction analysis of esophageal squamous-cell carcinoma. The results underscore the direct genetic contribution to esophageal squamous-cell carcinoma risk, and the genetic contribution to esophageal squamouscell carcinoma through interaction with alcohol consumption. There are other cancers could be taken genome-wide association study and genome-wide gene-environment interaction analysis to find the direct genetic contribution caused by the risk factors of cancer. 
Some GWAS researches have reported that the articles performed the genome-wide gene-environment interaction analysis in alcohol drinkers and emphasize the importance of further analyses of interactions between genetic variants and the specific environmental factors in high-risk populations. Because the gene-environment interaction of cancer is very complex. Any new esophageal squamouscell carcinoma susceptibility loci cannot be considered only one risk factor, for example, the alcohol drinking. The gene susceptibility loci of cancer may be caused by environmental agents, chronic infection, et al., including alcohol drinking. The risk factors attributable fraction of susceptibility loci should be calculated. The risk factors attributable fraction of susceptibility loci means how much every risk factor of cancer contributing on the cause of susceptibility loci.

There are lots of risk factors of cancer which are not limited on alcohol consumption. It is valuable to take on further research on these risk factors of cancers including esophageal squamous-cell carcinoma. Just like the research of Wu et al., ${ }^{6}$ and other GWAS researches, we can take on genome-wide association analyses of esophageal squamous cell carcinoma and other cancers to identify susceptibility loci, the gene-risk factors interactions and the risk factors attributable fraction of susceptibility loci. As the carcinogenesis theory of gene multiple hits which Han-You ${ }^{13}$ has researched and published considered cancer is caused by the environmental pollution agents and other gene multiple hits. As referenced before, lots of GWAS researchers have found the genetic etiology of multiple hits, the multiple gene susceptibility loci. We could create the novel way to prevent and treat the cancer from the view points of the genetic etiology of multiple hits, multiple susceptibility loci by intervening the risk factors of cancer.

Firstly, we should pay more attention to the environmental pollution agents, including more than 30 occupational agents have been identified as carcinogens by IARC. If we find the risk factors of cancer have gene-risk factors interactions, identify their gene susceptibility loci, interaction pathway and the risk factors attributable fraction of susceptibility loci. We could progress on more effective prevention of cancer. Furthermore, creations of chemicals or drugs of the isomeride or changed structure of cancer risk factors to prevent or treat the cancers caused by the highest risk factors of cancer attributable fraction of susceptibility loci could be come into fact. Especially the more than 30 occupational agents have been identified as carcinogens by IARC, their gene susceptibility loci and interaction pathway may be simple and easy to define. But they may be the highest risk factors of cancer attributable fraction of susceptibility loci. The occupational agents and environmental agents usually have clear structures. The creation of the anti-chemicals of occupational agents and environmental agents, this is the blocker, inhibitors or the blocking agent of the cancer risk factors, may be easy when they interact with gene. So the creations of chemicals or drugs to prevent or treat the cancers caused by the highest risk factor of cancer attributable fraction of susceptibility loci could be easy.

Preventing or treating cancer caused by occupational agents and environmental agents could result in possible creation of drugs. Preventing and treating cancer caused by the tobacco smoking, alcohol drinking, infectious agents and other carcinogens including physical factors could also result in possible creation of drugs after genomewide association analyses of the cancer to identify susceptibility loci and the gene-risk factors interactions have been done and have found their interaction pathways when they are the highest risk factors of cancer attributable fraction of susceptibility loci.

\section{Discussion}

As the environment pollution and climate change have not been controlled but something of more and more severe. And climate change may create new pollution agents. Cancer control may be more difficulty in the environment of continuing pollution and climate change. So the intervention, prevention and treatment or even cure of the cancer by blocking, inhibiting the gene-risk factors of cancer interactions are much too useful. The strategy and the new concept to create new drugs for preventing and treating cancer by blocking, inhibiting the gene-risk factors of cancer interactions has not been done or reported up to now. Comparing with the tradition method of cancer treatment, the chemicals therapy, radiation, operation, biotechnology and their combinations, the new method by blocking, inhibiting the gene-risk factors of cancer interactions must be modern and novel. The character of the new method is to treat or cure the cancer by intervention and prevention. Which is based on the gene multiple hits theory of carcinogenesis, modern cancer etiology and modern molecular technologies. So the mechanism of the novel process to create drugs for cancer prevention and treatment is scientific and acceptable.

\section{Conclusion}

This article reported a novel process to create drugs for cancer prevention and treatment by modern molecular technology. The creations of chemicals or drugs of the isomeride or changed structure of cancer risk factors to prevent or treat the cancers caused by the highest risk factors of cancer attributable fraction of gene susceptibility loci could be come into fact. This is the creation of blockers, inhibitors or the blocking agent of the cancer risk factors to prevent and treat cancers may be easy when intervening the risk factors of cancer interacting with genes. The strategy and the new concept to create new drugs for preventing and treating cancer by modern cancer molecular research technology, the genome-wide association study, the gene-risk factors of cancer interactions, definition of exposure of the risk factors of cancer and the carcinogenesis theory of gene multiple hits, could have great value to pursue and research further. But much research work and investigation should be done before she is to come into fact. The report may direct the new aspect of research for cancer prevention and treatment.

\section{Acknowledgments}

None.

\section{Conflicts of interest}

Author declares that there is no conflicts of interest.

\section{References}

1. Hashibe M, Mckay JD, Curado MP, et al. Multiple $A D H$ genes are associated with upper aerodigestive tract cancers. Nat Genet. 2008;40(6):707-709.

2. McKay JD, Truong T, Gaborieau V, et al. A genome-wide association study of upper aerodigestive tract cancers conducted within the INHANCE consortium. PLoS Genet. 2011;7(3):e1001333.

3. Cui R, Kamatani Y, Takahashi A, et al. Functional variants in $A D H 1 B$ and $A L D H 2$ coupled with alcohol and smoking synergistically enhances esophageal cancer risk. Gastroenterology. 2009;137(5):1768-1775. 
4. Wang LD, Zhou FY, Li XM, et al. Genome-wide association study of esophageal squamous cell carcinoma in Chinese subjects identifies susceptibility loci at PLCE1 and C20orf54. Nat Genet. 2010;42(9):759_ 763.

5. Abnet CC, Freedman ND, Hu N, et al. A shared susceptibility locus in $P L C E 1$ at 10q23 for gastric adenocarcinoma and esophageal squamous cell carcinoma. Nat Genet. 2010;42(9):764-767.

6. Wu C, Kraft P, Zhai K, et al. Genome-wide association analyses of esophageal squamous cell carcinoma in Chinese identify multiple susceptibility loci and gene-environment interactions. Nat Genet. 2012;44(10):1090-1097.

7. Sun T, Miao X, Zhang X, et al. Polymorphisms of death pathway genes FAS and FASL in esophageal squamous-cell carcinoma. J Natl Cancer Inst. 2004;96(13):1030-1036.

8. Zhang X, Miao X, Tan W, et al. Identification of functional genetic variants in cyclooxygenase-2 and their association with risk of esophageal cancer. Gastroenterology. 2005;129(2):565-576.

9. Sun T, Gao Y, Tan W, et al. A six-nucleotide insertion-deletion polymorphism in the CASP 8 promoter is associated with susceptibility to multiple cancers. Nat Genet. 2007;39(5):605-613.
10. Lewis SJ, Smith GD. Alcohol, ALDH2, and esophageal cancer: a meta-analysis which illustrates the potentials and limitations of a Mendelian randomization approach. Cancer Epidemiol Biomarkers Prev. 2005;14(8):1967-1971.

11. Hiyama T, Yoshihara M, Tanaka S, et al. Genetic polymorphisms and esophageal cancer risk. Int J Cancer. 2007;121(8):1643-1658.

12. Akbari MR, Malekzadeh R, Shakeri R, et al. Candidate gene association study of esophageal squamous cell carcinoma in a high-risk region in Iran. Cancer Res. 2009;69(20):7994-8000.

13. X. Han-You. The new theory of carcinogenesis-The theory of gene multiple hits. Annals of Oncology. 2008;19(Suppl 1):i53-i54.

14. Knudson AG. Heredity and human cancer. Am J Pathol. 1974;77(1):7784.

15. IARC Monographs on the Evaluation of Carcinogenic Risks to Humans. Vol. 1-91, Lyon, France: International Agency for Research on Cancer; 1972-2007.

16. IARC Handbooks of Cancer Prevention. Vol. 1-11. Lyon, France: International Agency for Research on Cancer; 1997-2007.

17. Kelsey JL, Gammon MD, John EM. Reproductive factors and breast cancer. Epidemiol Rev. 1993;15(1):36-47. 